\title{
HANDHELD VOLUMETRIC SCANNER FOR 3D PRINTED INTEGRATIONS OF HISTORICAL ELEMENTS: COMPARISON AND RESULTS
}

\author{
D. De Luca $^{1}$, M. Del Giudice ${ }^{1}$, N. Grasso ${ }^{2}$, F. Matrone ${ }^{2, *}$, A. Osello ${ }^{1}$, M. Piras ${ }^{2}$ \\ ${ }^{1}$ Department of Structural, Geotechnical and Building Engineering (DISEG), Politecnico di Torino, Corso Duca degli Abruzzi 24, \\ 10129 Torino, Italy - (daniela.deluca, matteo.delgiudice, anna.osello)@polito.it \\ ${ }^{2}$ Department of Environment Land and Infrastructure Engineering (DIATI), Politecnico di Torino, Corso Duca degli Abruzzi 24, \\ 10129 Torino, Italy - (nives.grasso, francesca.matrone, marco.piras)@ polito.it
}

\author{
Commission II, WG II/8
}

KEY WORDS: Point clouds, handheld scanner, structured light, digital model, 3D printing, restoration, Cultural Heritage

\begin{abstract}
:
The latest technologies in the field of geomatics and additive manufacturing can provide a significant support to the restoration and conservation activities of Cultural Heritage $(\mathrm{CH})$. In particular, the recent developments for both the 3D scanning techniques and the $3 \mathrm{D}$ printing systems are able to speed up the survey and the reconstruction of historical fragments gone lost. This research compares different meshes, obtained with different instruments and techniques, in particular a medium and a short- range volumetric handheld camera, with the aim of evaluating the best solution for the 3D printing and provide some guidelines for this kind of operation. Therefore, the focus is mainly on identifying the most effective solution to describe, represent and subsequently model small architectural details in the most automatic way, in order to step from the survey to the final printed result in the shortest time possible. Moreover, an attempt to integrate and complete not only sculptural details, by now well stated in the state of art, but also architectural elements, respecting the principles of reversibility and material recognisability typical of the modern restoration theories, has been done. The case study examined is an historical wooden gilded door with the lack of some decorative parts, in a church in the centre of Turin (Italy), symbol of the baroque architecture and damaged by a fire occurred during the Second World War.
\end{abstract}

\section{INTRODUCTION}

In the last years the integration of 3D surveying, modelling and printing for restoration purposes of Cultural Heritage $(\mathrm{CH})$ objects has become an increasingly widespread and applied methodology.

In this framework, the modern theories of restoration (Restoration Chart, 1972), which tend to prefer the use of different materials from the originals, to make the intervention explicit and recognizable, and that opt for actions that are as reversible as possible, make three-dimensional printing an excellent solution to problems of gaps or integration of missing fragments. In fact, the possibility of printing ad hoc pieces starting from a digital model, in a variety of different materials, makes this technique very versatile and easily reproducible.

Moreover, considered the numerous interventions that the $\mathrm{CH}$, and in particular the ecclesiastical buildings, would need in Italy, it is appropriate to also consider low-cost solutions and, in some cases, prefer them to those more accurate and faithful to the original, but certainly more expensive.

In particular, the case study here proposed envisages the integration of three decorative parts of an XVIII century wooden door, in a church in the centre of Turin (Italy). Specifically, this church suffered war damage in 1943, following which a fire destroyed the choir (with decorations, furnishings and archives) and all the wooden sculptures of the altar, leaving still today obvious signs of carbonation in many parts.

Precisely for this reasons, the proposed solution provides, on one hand, the 3D printing of these decorative elements with materials clearly different from the original and, on the other, with characteristics that nevertheless remember its history.
Therefore, the material used for the printed integration is the black polylactic acid (PLA), in order to recall the fire as well as the other historical carbonated parts, already blackened, and to be distinctly contemporary.

In similar studies (Hayes et al., 2015; Tucci and Bonora, 2007), in addition to $3 \mathrm{D}$ printing, it was also decided to use the subtractive fabrication techniques, as CNC machining. Despite the many advantages, such as the ability to model parts with complex shapes without compromising precision requirements (Tucci and Bonora, 2011) and the wide range of reproducing materials (Scopigno et al., 2017), this solution was not chosen because more expensive, if compared with the $3 \mathrm{D}$ printing, and because of the mayor amount of wasted material, from a sustainable and ecological point of view. In addition, the increasing number of $3 \mathrm{D}$ printers, as well as online printing services, and the extreme easiness of reproducibility were a determining factor for the client to opt for additive manufacturing.

Another factor to be considered is the limited time for accessing to the case study. In fact, since today the church is closed and occasionally opened by volunteers, it was decided to test a methodology that compares not only the accuracy of the results obtained, but also the times of acquisition and processing. Therefore, although also traditional systems as the TLS were employed, handheld 3D scanner systems have been used, allowing a first data processing in real-time, in order to be able to immediately control the data acquisition and evaluate the results. Besides, since one of the missing elements is in the lower part of the door, few centimetres from the floor, the 3D scanner systems were preferred also to the close range photogrammetry (CRP) which, in addition to requiring longer 
acquisition time, could not reach the lower, and almost hidden, parts of the decoration. For this reason, a photogrammetric acquisition of the entire portal was carried out for documentation purposes, but the individual elements were not investigated and compared with this technique as Boehler and Marbs (2004) and Weighert et al. (2019) did, since it was not even necessary to carry out a thorough texturing due to the black print.

Hence, this contribution tries to identify the most effective solution to describe, represent and subsequently model small architectural details in the most automatic way, in order to step from the survey to the final printed result in the shortest time possible. Moreover, an attempt to integrate and complete architectural elements, respecting the principles of reversibility and material recognisability, has been done.

A description of the procedures for creating, through 3D printing, detailed replicas of historical objects is therefore proposed. Two methods will be tested:

- the first exploits the geometries obtained from the point clouds, then it analyzes them in the software for parametric digital modeling;

- the second, directly analyzes the surfaces in the 3D printing software.

In conclusion, the results of these two procedures will be explained and the requirements for obtaining good quality printing will be defined.

\subsection{Related works}

Today the use of 3D printing is mainly applied in industry for the prototyping of small objects to be used on site, however, thanks to its potentialities, it is possible to extend its use also in the building and $\mathrm{CH}$ sector.

In this field, in addition to the conservation and restoration issues, it really interesting to cite the educational purposes, where it is possible to experiment new techniques for the reproduction of $\mathrm{CH}$ elements. In fact, many museums are using these instruments to realize precise and detailed models with innovative materials. Moreover, thanks to these tools it is also possible to guarantee the conservation, usability and replicability of cultural goods without damaging them (Balletti et al., 2017).

In addition, users can understand the object not only through the use of sight, but can live a multisensorial experience using touch as well (Ballarin et al., 2018; Capurro et al., 2014; D'Agnano et al., 2015), even if it has to be considered that, in most cases, this type of experience is not feasible because the objects on display are flimsy, damaged or with a strong historical value to be stored in protected places (Pieraccini et al., 2001).

The advantage of replicating an element, through the new 3D printers, becomes the opportunity to define standards and methods of surface generation useful for prototyping too (Xu et al., 2017) and to define standard guidelines ( $\$ 2.3$ ). Production costs are reduced and it is possible to create digital libraries of objects that can be used for a large number of users. Within the process of digitizing information and in the three-dimensional representation of objects, Building Information Modelling can support the creation of digital databases shared between multiple users with different levels of information (Osello, 2012), also in the case of small architectural objects. They could be inserted into a building database with different Level of Detail or directly with the mesh, providing more in-depth information.

\subsection{Case studies}

The case studies proposed are some wooden fragments, destroyed by a fire, as aforementioned, and belonging to an historical wooden gilded door in the Church of Santissima Trinità (Turin), that dates back to the XVII century and that is a symbol of the baroque architecture (Figure 1).

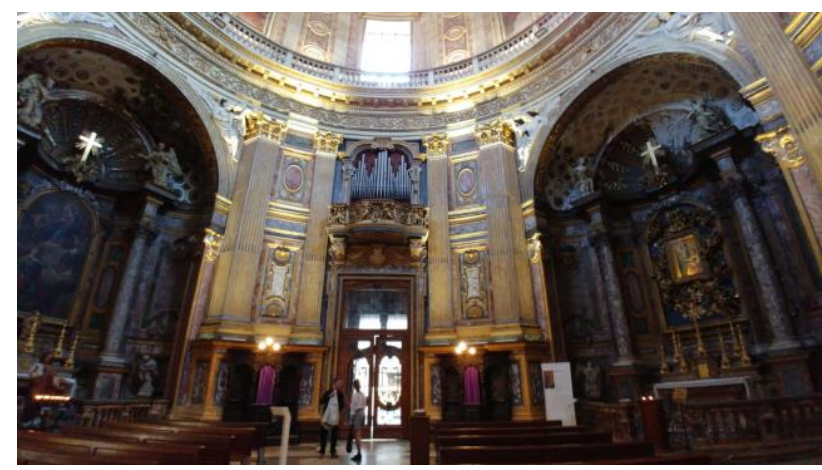

Figure 1. Interna part of the Church of the Santissima Trinità.

On the side of the main altar of the Church, there are two symmetrical doors (Figure 2), richly decorated, that provide the access to the service areas. In particular, the one on the left side is the reference (Figure 3a), while the one on the right has the missing elements (Figure $3 b$ ).

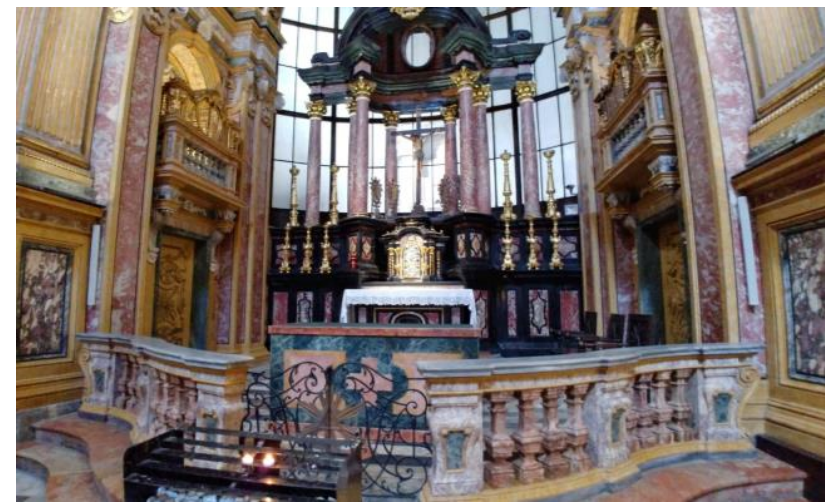

Figure 2. The two symmetrical doors on the side of the altar.
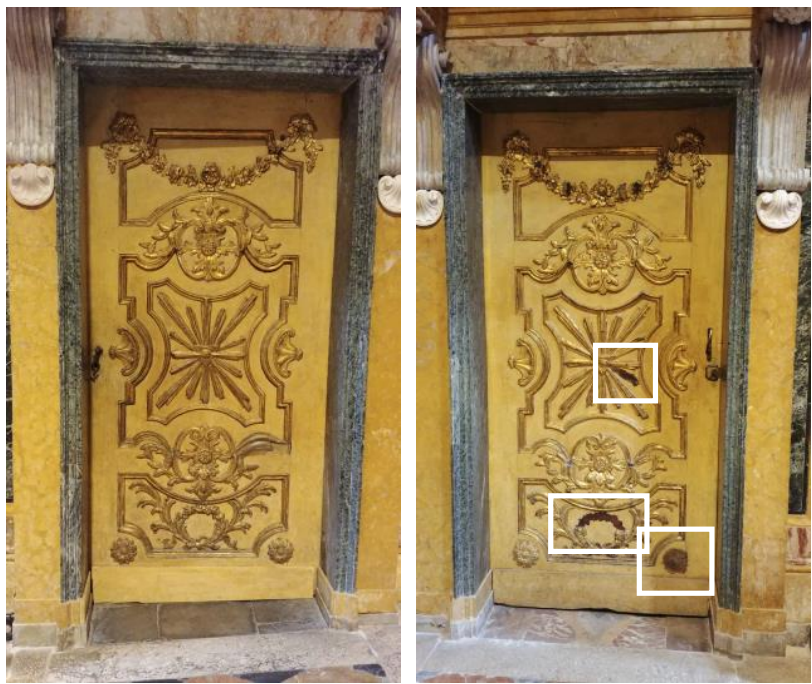

Figure $3 \mathrm{a}$ and $\mathrm{b}$. The reference door used for the survey (left) and the ruined one (right) with the identification of the missing elements. 
The survey was carried out mainly on the intact and complete portal in order to allow an accurate reconstruction of the fragments based on the original geometries, but also the one with the missing parts has been surveyed to have a correct support for the 3D printing. In particular, the decorative parts missing are a branch of a wreath of flowers (Figure 4), a sunbeam (Figure 5) and a decorative rosette (Figure 6).
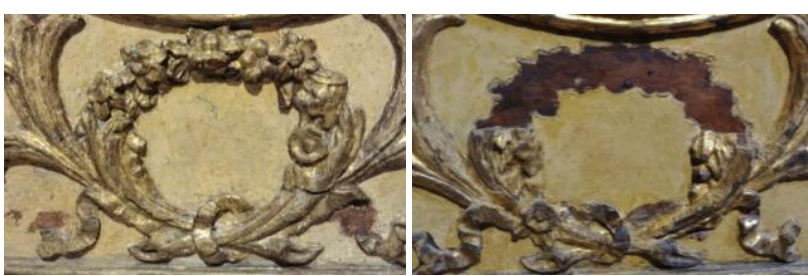

Figure 4. Branch of a wreath of flowers.

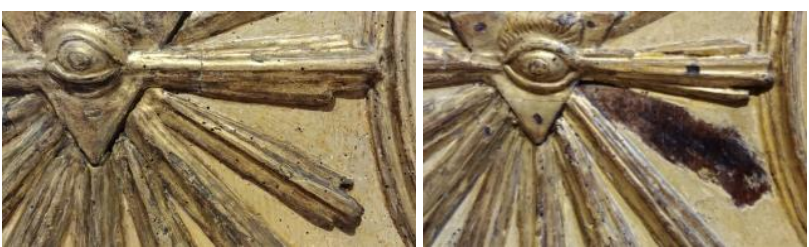

Figure 5. Sunbeam.

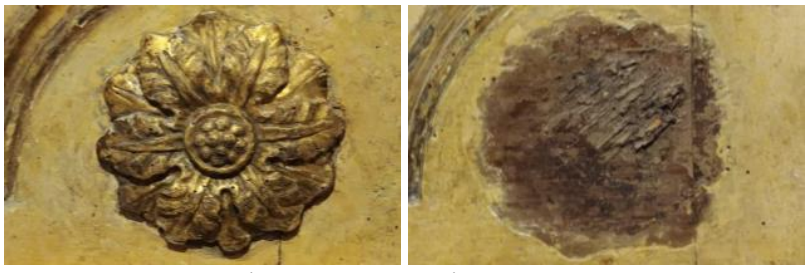

Figure 6. Decorative rosette

\section{METHODOLOGY}

The workflow for the generation of the replicas begins with the data acquisition and processing of the meshes, through the point clouds, and ends with the 3D model validation and printing.

Concerning the data acquisition, even if already known that the structured light 3D scanner systems would have given better results than the other techniques employed, we anyway decided to carry out a comparison between the generated meshes, to assess which level of detail was appropriate for 3D printing, considering also the triangulation degree of simplification due to the inaccuracy of the printing.

\subsection{Data acquisition}

The data acquisition involved four different instruments and techniques:

- a Terrestrial Laser Scanner FARO CAM2 Focus 3D for the laser scanning point cloud (Figure 7a) where 3 scans have been made

- a SONY ILCE-5100 for the photogrammetric survey

- two handheld scanners for the structured light acquisition, a medium and a short-range.

In this framework, the use of the handheld structured light 3D scanners F6 SMART (medium range) and SR (short range) (https://mantis-vision.com/professional-solutions/f6-smart about/) whose specifications could be seen in Table 1, enables a high level of detail with a shorter time of acquisition and postprocessing. In fact, the positioning of markers for the frame registration is not necessary and the acquisition span is relatively fast. This instrument, with a NIR and RGB camera sensor, has been equipped with a HP backpack PC desktop (16 Gb RAM and NVIDIA® Quadro® P5200 GPU) and a monitor, in order to simultaneously view the acquisition in $2 \mathrm{D}, 3 \mathrm{D}$ or in the IR field and process the data (Figure 7b).

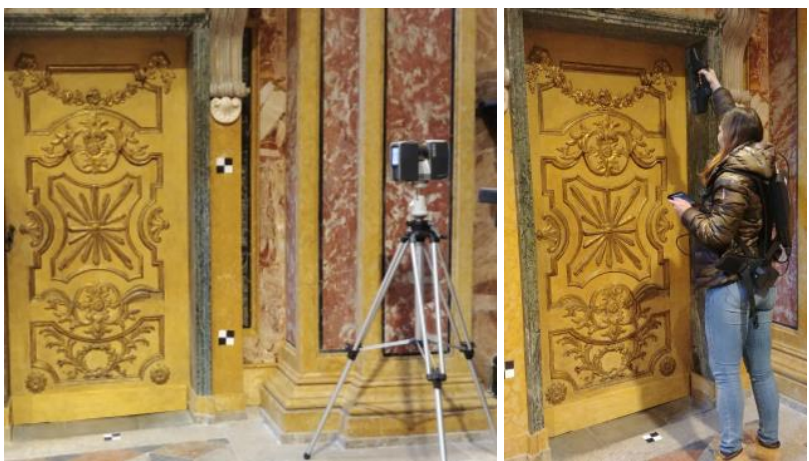

Figure 7a and $\mathrm{b}$. TLS and F6 SMART data acquisition.

\begin{tabular}{|l|l|l|l|}
\hline & $\begin{array}{l}\text { FARO } \\
\text { Focus 3D }\end{array}$ & F6 SMART & F6 SR \\
\hline Accuracy & $\begin{array}{l}\text { @ } 10 \mathrm{~m}: \\
0,3 \mathrm{~mm} *\end{array}$ & up to $0,5 \mathrm{~mm}$ & up to $0,1 \mathrm{~mm}$ \\
\hline Depth of field & $0,6-130 \mathrm{~m}$ & $0,5-4.5 \mathrm{~m}$ & $0,25-0,75 \mathrm{~m}$ \\
\hline Field of view & $\begin{array}{l}\text { @ } 0,5 \mathrm{~m}: \\
510 \times 670 \mathrm{~mm} \\
\text { @ 4,5 m: } \\
4585 \times 6070 \mathrm{~mm}\end{array}$ & $\begin{array}{l}\text { @ } 0,25 \mathrm{~m}: \\
245 \times 345 \mathrm{~mm} \\
\text { @ 0,75 m: } \\
735 \times 1030 \mathrm{~mm}\end{array}$ \\
\hline $\begin{array}{l}\text { Angular field } \\
\text { of view }\end{array}$ & $300^{\circ} \times 360^{\circ}$ & $54^{\circ} \times 68^{\circ}$ & $54^{\circ} \times 68^{\circ}$ \\
\hline $\begin{array}{l}\text { Color camera } \\
\text { resolution }\end{array}$ & $\begin{array}{l}\text { up to } 70 \\
\text { Mpix }\end{array}$ & $1,3 \mathrm{MPix}$ & $1,3 \mathrm{MPix}$ \\
\hline $\begin{array}{l}\text { Video frame } \\
\text { rate }\end{array}$ & $97 \mathrm{FPS}$ & $8 \mathrm{FPS}$ & $8 \mathrm{FPS}$ \\
\hline Weight & $5,2 \mathrm{Kg}$ & $1 \mathrm{Kg}$ & $1 \mathrm{Kg}$ \\
\hline Cost & $30.000 €$ & $9.000 €$ & $9.000 €$ \\
\hline & $*$ without filtering @ 90\% rfl.
\end{tabular}

Table 1. Instruments specifications.

\subsection{Data processing}

The data processing took place in two stages: the first and fastest one was carried out on site, to verify the correctness of the data acquired through the two F6 (with the point cloud generation in real time and the first processing algorithms), the second and more accurate one was afterwards conducted in the laboratory (Figure 8).

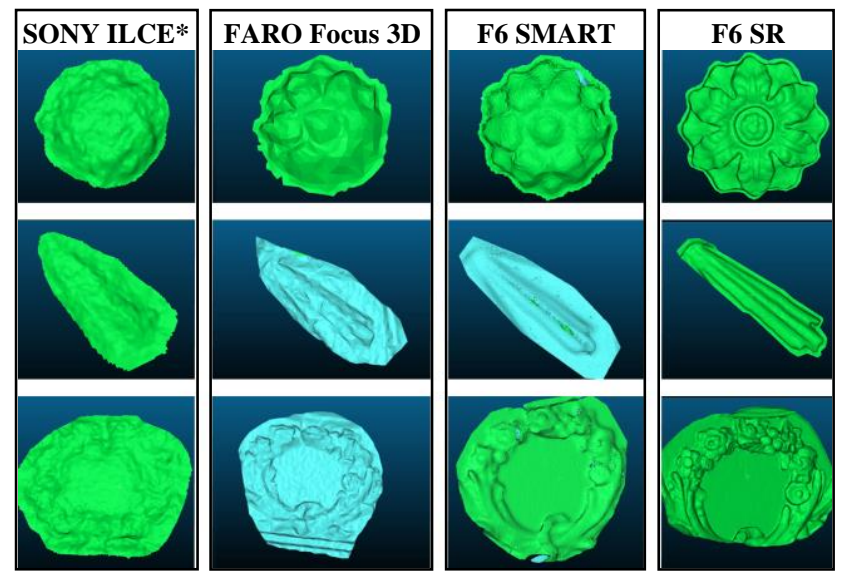

*The SONY result has not to be compared with the other, as no CRP has been conducted.

Figure 8. In the column the meshes of the 3 fragments acquired with the different instruments. 
The result with the highest level of detail was achieved with the F6 short range (Table 2), although acquisition and processing times were slightly higher than those of the F6 medium range (Table 3).

The following specifications concern the decorative rosette.

\begin{tabular}{|c|c|c|c|}
\hline & TLS & $\begin{array}{c}\text { F6 medium } \\
\text { range }\end{array}$ & $\begin{array}{c}\text { F6 short } \\
\text { range }\end{array}$ \\
\hline n. points & 556 & 5262 & 414.829 \\
\hline n. triangles & 1.028 & 10.161 & 826.970 \\
\hline n. frames & & 251 & 1.261 \\
\hline
\end{tabular}

Table 2. Mesh properties of the decorative rosette.

\begin{tabular}{|c|c|c|c|}
\hline & TLS & $\begin{array}{c}\text { F6 medium } \\
\text { range }\end{array}$ & $\begin{array}{c}\text { F6 short } \\
\text { range }\end{array}$ \\
\hline Data acquisition & $24 \mathrm{~min}$ & $31 \mathrm{sec}$ & $2,5 \mathrm{~min}$ \\
\hline $\begin{array}{c}\text { Data processing } \\
\text { on site } \\
\text { (point cloud) }\end{array}$ & $/$ & $2,5 \mathrm{~min}$ & $6 \mathrm{~min}$ \\
\hline $\begin{array}{c}\text { Data processing } \\
\text { in the } L a b \\
\text { (point cloud) }\end{array}$ & $12 \mathrm{~min}$ & $4 \mathrm{~min}$ & $13 \mathrm{~min}$ \\
\hline $\begin{array}{c}\text { Data processing } \\
\text { in the } L a b \\
\text { (denoise) }\end{array}$ & $13 \mathrm{~min}$ & $11 \mathrm{~min}$ & $17 \mathrm{~min}$ \\
\hline $\begin{array}{c}\text { Data processing } \\
\text { in the } \text { Lab } \\
\text { (mesh) }\end{array}$ & $6 \mathrm{~min}$ & $7 \mathrm{~min}$ & $9 \mathrm{~min}$ \\
\hline
\end{tabular}

Table 3. Time comparison for obtaining the mesh of the decorative rosette through the Echo software of Mantis Vision.

Once obtained the meshes, they were compared with each other, keeping the F6 SR mesh as reference.

For the comparison, a range between $1 \mathrm{~mm}$ and $5 \mathrm{~mm}$ has been set. On the left side there is the overlap of the two meshes (the reddish is the F6 SR reference), while on the right side there is the distance computation and the grey colour indicate the areas where the distance is higher than $5 \mathrm{~mm}$.

In Figure 9, F6 vs. TLS, it can be noticed that the deviations are greater in the lower parts of the decoration, in fact, as the TLS was positioned at about $1,7 \mathrm{~m}$ from the ground, it was not possible to optimally acquire those data towards the floor.
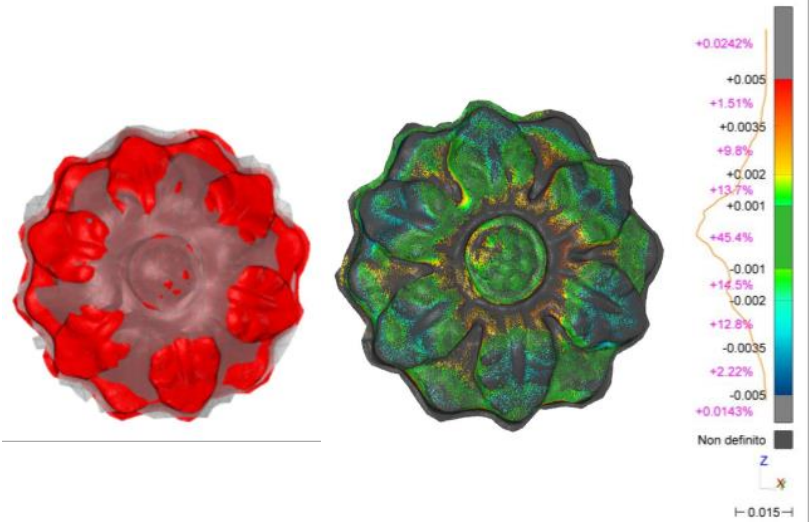

Figure 9. On the left, comparison between the meshs of TLS (grey) and F6 short range (red); on the right, distance computation.

In the comparison with the two F6 meshes (Figure 10) the overlapping is higher, but still some details are lost. Also the distance is globally lower, but not satisfactory.
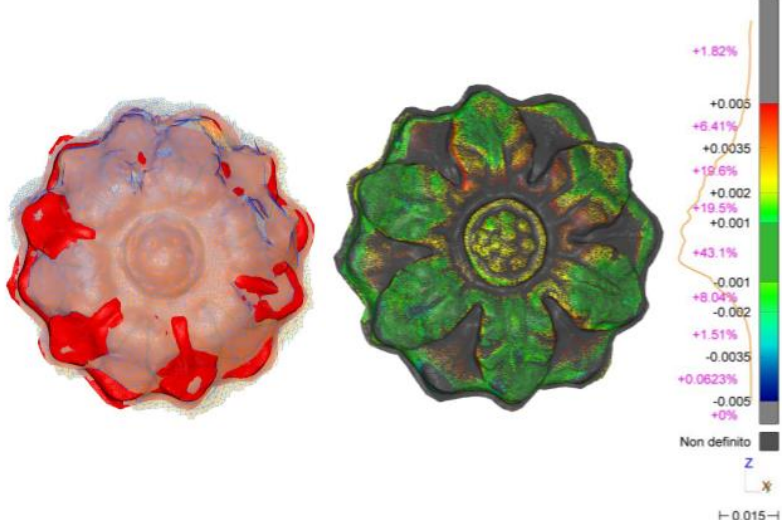

Figure 10. Comparison between the meshs of F6 medium range (yellow) and F6 short range (red).

\subsection{Creation of the digital information model}

At this point, even if a good quality mesh was already obtained, a new three-dimensional digital model was generated through different methodologies, in order to evaluate which one could suit better for the final print.

The first method allows creating the geometries through the import of the point cloud into a software for digital modeling as Revit. This method do not use any automation for 3D modeling as it involves only user's skills in creating the geometries, taking into account each time simplifications of the shapes. Even if inserting the mesh into an object-oriented software is not a correct process for the 3D printing, it permits to store not only geometric data but also to insert alphanumeric properties able to populate a digital archive that is always accessible. In this way, it is possible to include in 3D architectural models, as the Building Information Model (BIM), some descriptive parameters useful to catalogue the mapped object and integrate it inside specific libraries. In addition, the advantage of creating BIM objects, ensures the editable geometry and alphanumeric part through the direct insertion of cards and schedules that reflect the items of the state of preservation of the object and any ongoing restorations.

So, the informative database can be shared and viewed by users without compromising the uniqueness of the data.

There is another method, which combines the information database of the BIM model with the correct three-dimensional representation of surfaces. The BIM model is based on a digital representation of physical and functional characteristics of surfaces. It is possible to integrate the geometric properties with specific software that convert the point cloud into mesh (Eastman et al., 2008).

On the other hand, the second methodology outlines the possibility of using software able to directly convert the point cloud into solid surfaces. Thanks to specific settings, a good level of surface detail can be achieved. To date, however, this method is not yet free of criticism. For these reasons, the test of different platforms seems to be the most suitable strategies to optimize the printing process. Through the use of digital models and with specific software it is possible to check the geometries and make the replica as similar as possible to the real one. Finally, thanks to the interoperability it is possible to study the best method to print the model and choose the best software. Thanks to these new important technologies, digital models are increasingly diversified and can be integrated with each other. In order to obtain an information model that can store data over time, the mapping of objects must be populated also with the point clouds. 
Therefore, the parametric digital model, created in this way, represents a virtual 3D model, accessible and editable over time, integrating information useful for the management and analysis of the state of preservation (Brumana et al., 2013).

\subsection{Generation of the mesh for the 3D printer}

Based on the data processed during the first phase of survey, the creation of solid surfaces has highlighted the need to test different softwares, indicating a series of critical points of the input data. The main platforms analyzed to generate the geometries from the survey data are: Cloud Compare, MeshLab, Geomagic Wrap, Reconstructor, Rhino-Meshflow.

By processing the files in the individual software the gaps within the point cloud can be highlighted, especially in the lower part of the portal. In this way, analyzing in detail only individual elements the achievement of an high degree of reliability of the digital model resulted difficult.

The first software analyzed is Reconstructor: importing the cloud generated by laser scanners, the surfaces can be directly obtained by clicking on the icon. Before finishing the operation, it is important to set the quality of the triangulations and the softness of the cloud (Figure 11). The result obtained shows strong gaps due to the lack of points in the cloud generated by the laser scanner
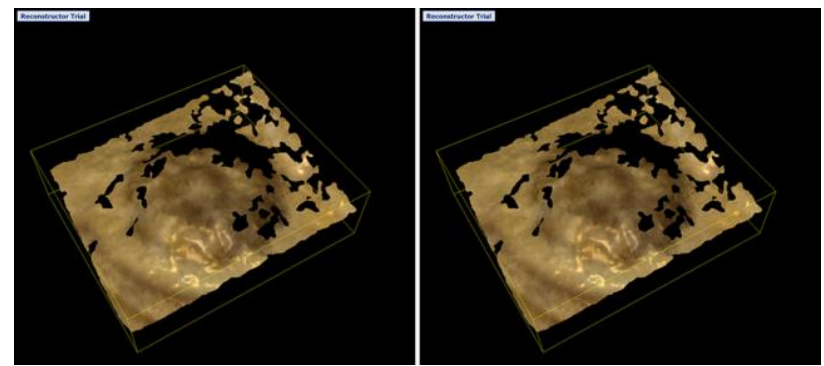

Figure 11. Creating mesh into Reconstructor

The second software tested is Geomagic Wrap: in this case by inserting the 3 point clouds obtained from the 3 different survey instruments, it is possible to recreate the surfaces setting the maximum number of triangulations and the quality of polygons. Unfortunately, even in this case, by varying the settings, the result obtained is not a high quality mesh. In fact, in addition to the too many missing triangles, the entire surface is not homogeneous, losing even the curved details and object recesses. The software has also the possibility to refine the 3D surface 3D obtained, changing the number of edges. Despite this further revision, the result achieved is not optimal for $3 \mathrm{D}$ printing (Figure 12).

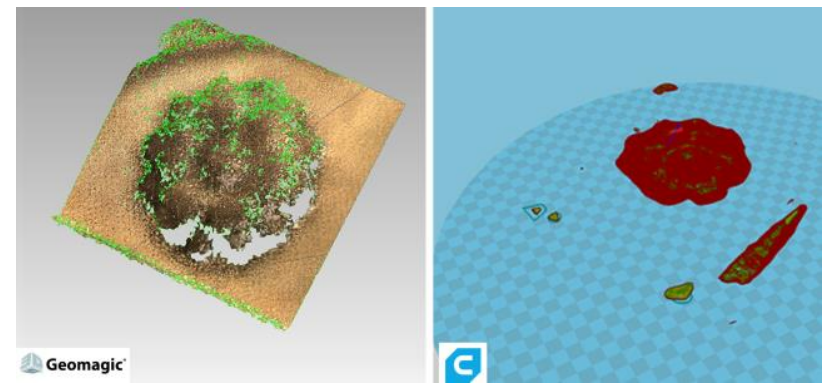

Figure 12. Creating mesh into Geomagic Wrap and check into CURA, the software for the $3 \mathrm{D}$ printing.
Another program examined is Rhinoceros with the Meshflow plug-in (Figure 13). In this case, the level of detail achievable is higher than in the previous applications, but it still contains imperfections.

It guarantees the possibility to set the distance from the points of the cloud and the accuracy used to generate the 3D geometry. A distinctive feature of this software is its interoperability with parametric digital modelling software, as it is possible to export the geometries in sat format.

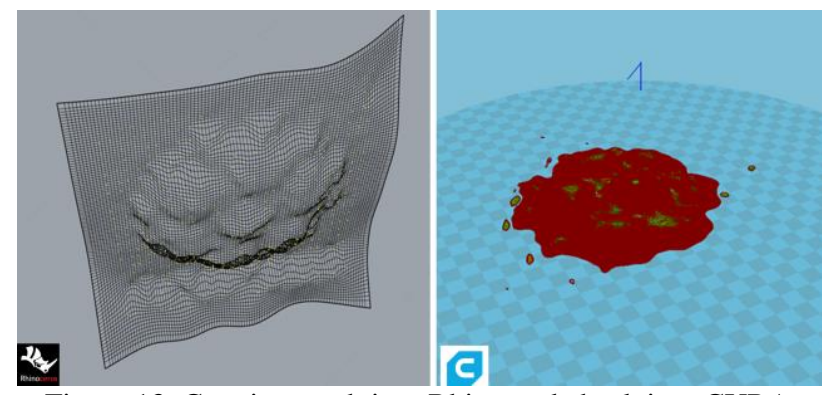

Figure 13. Creating mesh into Rhino and check into CURA.

Finally, the other two softwares tested are: MeshLab and CloudCompare. The first allows to set the number of polygons to be analyzed by inserting the cloud and selecting the region of interest. Through an algorithm inside the software it is possible to generate solid surfaces by entering the "grouping radius". In Figure 14 is shown the result of this process. The $3 \mathrm{D}$ surface generated, is quite a good result, especially with regard to the reconstruction of missing points, although the degree of reliability is not fully achieved because the level of detail is not so high.

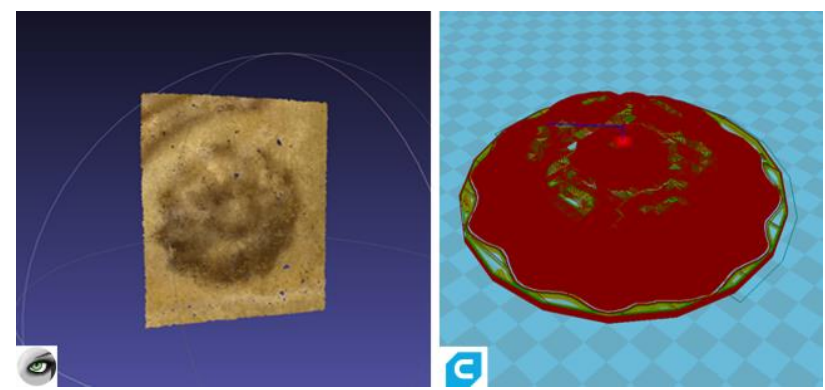

Figure 14. Creating mesh into MeshLab and check into CURA

The last software, similar to the previous one, enable to act directly on a specific region. Is possible to set the number of points to be analyzed, the depth of the elements and the accuracy of the position (Figure 15).

Analyzing the model on the 3D printing software it is clear a good surface smoothness and an acceptable level of detail.
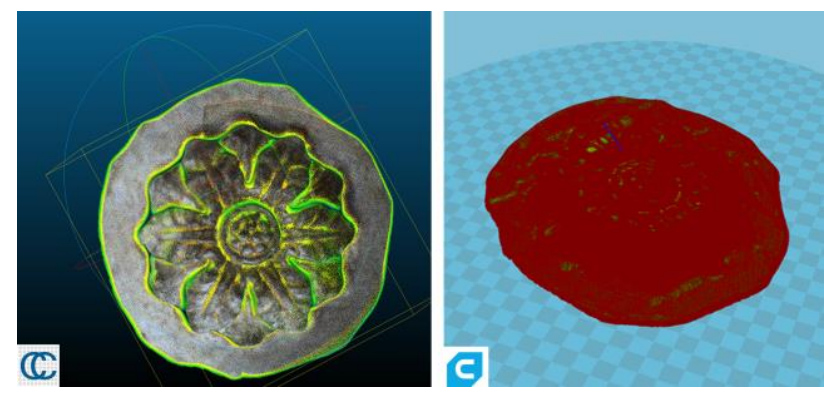

Figure 15. Creating mesh into Cloud Compare and check into CURA. 


\subsection{Parameters and process for 3D printing}

Once 3D surfaces with a proper quality are obtained, it is possible to check if the geometry is printed correctly using additional Meshmixer software. This program focuses to analyze the file in the Standard Triangulation Language (STL) format. This type of format is the most common in the traditional printing process, because it converts surfaces into more or less complex triangles (Hager et al., 2016). Using an intermediate program such as Meshmixer, the accuracy of the printed object increases because it acts on the way of filling the holes in three directions: filling the plate, the minimum amount and the removal of surface impurities. The higher the value, the less precise the geometry because the incorrect triangulations are subdivides into small parts. Once this additional check has been carried out, it is possible to create the .stl or .obj file to be imported to CURA (Dellosta, 2017). This additional software enables the conversion of the digital model into layers that will be printed (Figure 16).

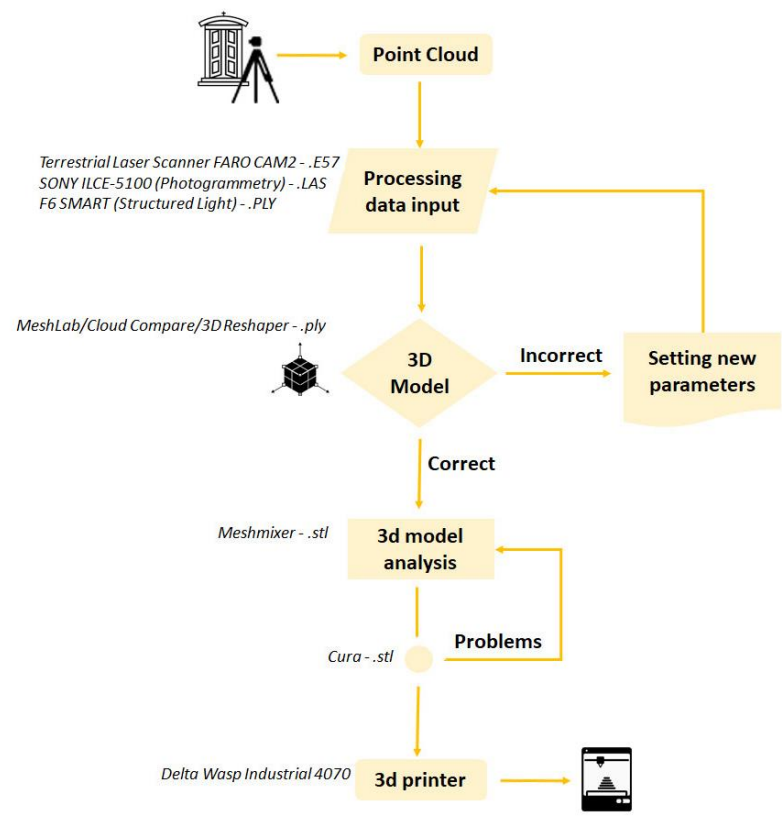

Figure 16. Process for data restitution.

The printer used is the Delta WASP Industrial 4070 (Figure 17), which has a maximum printing plate size of $40 \mathrm{~cm}$ and 70 $\mathrm{cm}$ in height.In this way the pieces to be reproduced can be easily printed in a single element without having to divide them. It took 3 hours to print the decorative rosette of $13 \mathrm{~cm}$ of diameter. The first settings to be inserted in the software concern the print quality you want to obtain. If the height of the print layer is low, the quality of the object increases significantly. This value, however, affects the printing time. In fact, with higher data the hours of printing increase.

Another important parameter to evaluate in order to obtain a smooth and flawless product is the thickness of the vertical walls or of the shell. The Fill Density parameter, or the filling of the layers, is also added to this value. To achieve an object more resistant to touch, it is useful to set the parameter with mediumhigh values in order to make the object lighter. The last settings to consider for a good print quality are the speed and temperature of the printer. The lower the speed, the greater the probability of obtaining a good result, while the temperature is linked to the type of material used. The printer provides the possibility of inserting multiple types of filaments; PLA, ABS and Nylon. The most common and resistant material is PLA, moreover it does not require high moulding temperatures and is economical, compared to ABS and Nylon (Polak et al., 2017).

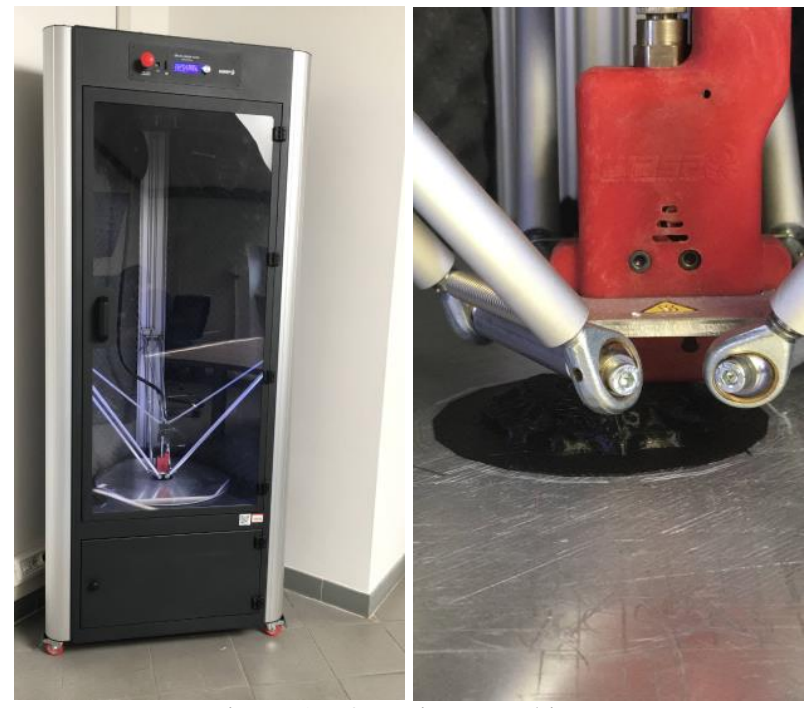

Figure 17.3D printer working.

Moreover, if there are projecting parts or elements that do not lie on horizontal planes, it is possible to check if they are correctly realized by means of special settings concerning the insertion of supports. They can be placed on the whole piece or only on the most delicate parts. In this way, the correct functioning of the printer is also managed, reducing vibrations that can damage the 3D product. Finally, to further refine the quality of the first print layer and to produce a more solid structure, it is possible to act on two parameters: Initial layer thickness and Cut off object bottom.

\section{RESULTS AND DISCUSSION}

\subsection{Interoperability}

In addition to the analyses previously carried out, it was necessary to analyze the input data formats.

From the Terrestrial Laser Scanner FARO CAM2, the formats accepted in the platforms above mentioned are: .las, .E57, .ply. By comparing the .las format in all the softwares, it is possible to highlight how it is not supported in MeshLab and Rhino. Reconstructor reads the file correctly but fails to process the points properly. Cloud Compare and Geomagic, on the other hand, besides reading the data correctly, guarantee a result that is not yet optimal but acceptable. Using the .E57 format, for MeshLab and Rhino the result is the same as the previous one. In this case, with the Reconstructor platform data was processed, but not exported for 3D printing, as this feature in the trial version is not activated. Finally, the only format that ensures that data is processed properly and the geometries exported correctly for 3D printing is .ply, with minor exceptions in the Geomagic and Rhino platforms (Table 4).

\begin{tabular}{|c|l|l|l|l|l|l|}
\cline { 2 - 7 } \multicolumn{1}{c|}{} & \multirow{2}{*}{ Format } & \multicolumn{5}{l|}{ Software } \\
\cline { 2 - 7 } & & CC & MeshL & Geom & Reconstr & Rhino/Mf \\
\hline \multirow{3}{*}{ TLS } & .las & yes & no & yes & no & no \\
\cline { 2 - 7 } & .E57 & no & no & no & no & no \\
\cline { 2 - 7 } & .ply & yes & yes & no & no & no \\
\hline
\end{tabular}

Table 4. Interoperability: Terrestrial Laser Scanner FARO CAM2 Focus 3D. 
The second instruments used for the survey are the portable scanners F6; in this case the cloud returned in las format does not guarantee an optimal reading of the data in the software, as it is not supported in MeshLab or Rhino. Instead, the format .E57, is read correctly even if with some inaccuracy in the Reconstructor platform, but even in this case the problem of the trial version does not allow to deepen the data reliability on the specific software for 3D printing. Instead, the .ply format, is the one that, also in this case, ensures good results (Table 5).

\begin{tabular}{|c|l|l|l|l|l|l|}
\cline { 2 - 7 } \multicolumn{1}{c|}{} & \multirow{2}{*}{ Format } & \multicolumn{4}{l|}{ Software } \\
\cline { 2 - 7 } & CC & MeshL & Geom & Reconstr & Rhino/Mf \\
\hline \multirow{3}{*}{ F\& } & .las & no & no & yes & no & no \\
\cline { 2 - 7 } & E57 & no & no & no & no & no \\
\cline { 2 - 7 } & .ply & yes & no & no & no & yes \\
\hline
\end{tabular}

Table 5. Interoperability F6 handheld scanners.

By testing these platforms, it is evident that some of them in the demo version do not permit to process the native file with a high grade of accuracy. The most interoperable format able to define a reliable 3D digital model is the .ply, in fact in almost all the platforms it is possible to read data and to export accurate solid surfaces. It is useful therefore to point out that the combination of laser scanner and .ply format seems to be the best solution.

To further test the interoperability between post processing and $3 \mathrm{D}$ printing software, the geometries obtained directly from the survey software were analyzed. The results highlight once again the need to test a software "bridge" to get the expected results. Unfortunately, with all the techniques used, it was not possible to print directly the generated 3D model, as it has discontinuity between the surfaces. While using surface control software such as Meshmixer, post-processing errors are greatly reduced.

\subsection{Guidelines}

Once interoperability and post-processing tests have been carried out on the point clouds, it is possible to define the correct procedure for the success of $3 \mathrm{D}$ printing. The most interoperable software today, which offers an excellent level of detail of individual products, among those tested, is Cloud Compare. It is possible to integrate the geometries in the BIM software, controlling once again the alphanumeric properties. Subsequently, the "bridge" software checks for any open surfaces, not detected previously, and exports the file in the .stl format. Then, after the final file has been obtained, within CURA it is optimal to set the height of the layer to $0,06 \mathrm{~mm}$, so as to print even the convex part. The thickness of the nozzle of the printer used is $0,7 \mathrm{~mm}$, so the value to be entered to homogenize the outer shell of the print must have a value equal to twice the nozzle. For the proposed case study, this parameter has been set to 1,4 millimeters and the density has been set to $30 \%$, in order to make the object stable in its original location, without further weighing it down (Figure 18).

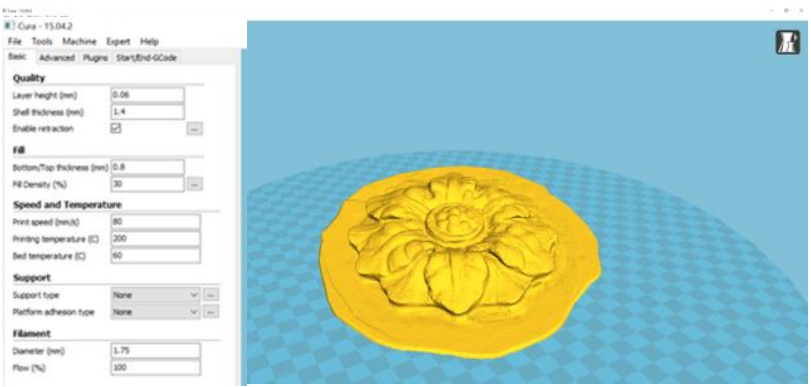

Figure 18. Setting parameters for 3D printing.
The printing speed is very low to limit smudges and inaccuracies. It is advisable to set it to $80 \mathrm{~mm} / \mathrm{s}$ with a temperature of 60 degrees of adhesion of the object to the printing plate. This ensures greater adhesion without damaging the bottom layer. In addition, the material used for printing is PLA, with a diameter of 1,75 $\mathrm{mm}$ in black (Figure 19).
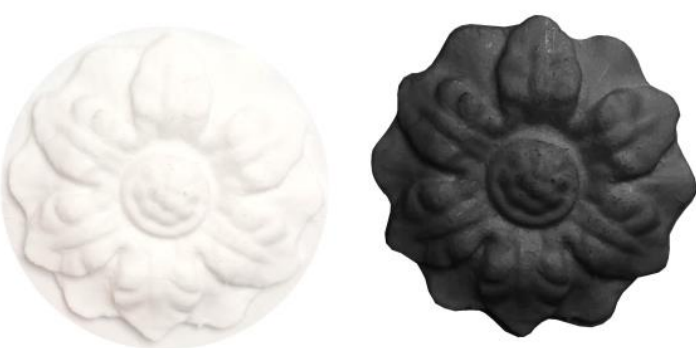

Figure 19. Printed object obtained with the described settings, white version for the test and black for the door integration.

\section{CONCLUSIONS}

In the end, it can be said that with the use of structured light handheld scanner it is possible to reproduce small or medium historical elements, that time and history have damaged and make them suitable to the sight and touch for educational and museum purposes with the 3D printing.

The shorter time of acquisition and the possibility to view the real-time point cloud are undoubtedly an added value to the already known maneuverability and lightness of these instruments.

Moreover, the adopted technology (opposing to the traditional handcrafting integration) demonstrates the possibility to reduce the costs of reproduction, but at the same time it respects the modern restoration principles.

Through this working methodology, it is also possible to carefully study partial reconstructions of the object. Using the integration of the geometries obtained with the survey of the pre-existing ones, a detailed 3D model is generated and perfectly carved in the parts that persist on the wooden door. In this way the reconstruction perfectly follows the state of conservation of the real element, without altering the historical and documentary value.

Thanks to the flexibility of the new process, it is possible to reproduce a copy of the object, modify it directly on the parametric software and realize it by the printer.

Finally, the versatility of the materials offered by the printers, the choice to use more or less extruders and precise settings, means that you can have more copies of detail to use at different times and places. Today printers allow achieving excellent results in few steps, but the limit still concerns the type of model and the type of survey carried out with the operator's expertise.

\section{ACKNOWLEDGMENTS}

Thanks to the Arch. Michele Ruffino for his help and continuous availability to open the Church for us, even with little notice.

Thanks also to the G4CH Lab of the DAD (Department of Architecture and Design) that lend us the F6 short range and to the Arch. Giacomo Patrucco for his advices on creating the meshes. 


\section{REFERENCES}

Ballarin, M., Balletti, C., Vernier, P., 2018. Replicas in cultural heritage: $3 \mathrm{D}$ printing and the museum experience. In: The International Archives of the Photogrammetry, Remote Sensing and Spatial Information Sciences, Vol. XLII-2, pp. 55-62. doi.org/10.5194/isprs-archives-XLII-2-55-2018

Balletti, C., Ballarin, M., Guerra, F., 2017. 3D printing: State of the art and future perspectives. Journal of Cultural Heritage. Elsevier, pp. 172-182.

doi.org/10.1016/j.culher.2017.02.10.

Boehler, W., Marbs, A., 2004. 3D scanning and photogrammetry for heritage recording: a comparison. Proc. 12th Int. Conf. on Geoinformatics - Geospatial Information Research: Bridging the Pacific and Atlantic, pp. 291-298.

Brumana R., Oreni D., Raimondi A., Georgopoulos A., Bregianni A., 2013. From survey to HBIM for documentation, dissemination and management of built heritage. Digital Heritage International Congress (Digital Heritage), pp. 497504.

doi: 10.1109/DigitalHeritage.2013.6743789

Capurro C.; Nollet D.; Pletinckx D., 2014. Tangible Interfaces for Digital Museum Applications: the Virtex and Virtex Light Systems in the Keys to Rome Exhibition. Proceedings of the 2015 Digital Heritage International Congress. Springer, New York.

D’Agnano F.; Balletti C.; Guerra F.; Vernier P., 2015. Tooteko: a Case Study of Augmented Reality for an Accessible Cultural Heritage, Digitization, 3D printing and Sensors for an Audiotactile Experience. In: The International Archives of the Photogrammetry, Remote Sensing and Spatial Information Sciences, Vol. XL-5/W4, pp. 207-2013.

Dellosta, M., 2017. Utilizzo delle nuove tecnologie per la diffusione della conoscenza - Stampa tridimensionale. BIM: verso Il catasto del futuro. Conoscere, digitalizzare, condividere. Il caso studio della Città di Torino, Gangemi Editore.

Eastman, C., Teicholz, P., Sacks, R., Liston, K., 2008. BIM HANDBOOK. A guide to Building Information Modeling for Owners, manager, Designer, engineers, and Contractors, Jonn Wiley \& Sons, Hoboken, New Jesey. doi.org/10.1002/9780470261309.ch5

Hager, I., Golonka, A., Putanowicz, R., 2016. 3D printing of buildings and building components as the future of sustainable construction. International Conference on Ecology and new Building materials and products, ICEBMP 2016. Procedia Engineering 151, Elsevier, pp. 292-299.

doi: $10.1016 /$ j.proeng.2016.07.357

Hayes, J., Fai, S., Kretz, S., Ouimet, C., White, P., 2015. Digitally-Assisted Stone Carving of a Relief Sculpture for the Parliament Buildings National Historic Site of Canada. In: ISPRS Annals of the Photogrammetry, Remote Sensing and Spatial Information Sciences, Vol. II-5/W3, pp. 97-103. doi:10.5194/isprsannals-II-5-W3-97-2015

Osello, A. 2012. Il futuro del disegno con il BIM per ingegneri e architetti, Dario Flaccovio Editore, pp. 29-33.
Pieraccini, M., Guidi, G., Atzeni, C., 2001. 3D digitizing of cultural heritage. Journal of Cultural Heritage. Elsevier, pp. 63-70.

doi.org/10.1016/S1296-2074(01)01108-6.

Polak R., Sedlacek, F., Raz, K., 2017. Determination of FDM printer settings with regard to geometrical accuracy. $28 \mathrm{TH}$ DAAAM international symposium on intelligent manufacturing and automation, DAAAM International, pp. 561-566.

doi: 10.2507/28th.daaam.proceedings.079

Restoration Chart, 1972. Available at http://www.sbappsaepi.beniculturali.it/getFile.php?id=783.

Scopigno, R., Cignoni, P., Pietroni, N., Callieri, M., Dellepiane, M., 2017. Digital Fabrication Techniques for Cultural Heritage: A Survey. Computer graphics forum, 36(1), pp. 6-21. doi.org/10.1111/cgf.12781

Tucci, G., Bonora, V., 2007. Application of high resolution scanning systems for virtual moulds and replicas of sculptural works. In: The International Archives of the Photogrammetry, Remote Sensing and Spatial Information Sciences, Vol. XXXVI-5/C53.

Tucci, G., Bonora, V., 2011. From real to... "real". A review of geomatic and rapid prototyping techniques for solid modelling in cultural heritage field. In: The International Archives of the Photogrammetry, Remote Sensing and Spatial Information Sciences, Vol. XXXVIII-5/W16, pp. 575-582.

doi:10.5194/isprsarchives-XXXVIII-5-W16-575-2011.

Xu, J., Ding, L., Love, Peter E.D., 2016. Digital reproduction of historical building ornamental components: from 3D scanning to $3 \mathrm{D}$ printing. Automation in Construction. Elsevier, pp. 8596.

doi.org/10.1016/j.autcon.2017.01.010.

Weighert, A., Dhanda, A., Cano, J., Bayod, C., Fai, S., Santana Quintero, M. 2019. A review of recording technologies for digital fabrication in heritage conservation. In: The International Archives of the Photogrammetry, Remote Sensing and Spatial Information Sciences, Vol. XLII-2/W9, pp. 773778 .

doi.org/10.5194/isprs-archives-XLII-2-W9-773-2019 